\title{
Efficiency of granulocytes and monocytes in breast cancer patients following radiotherapy in KSA.
}

\author{
Taghreed A. Hafiz ${ }^{1}$, Aminah A. Alghamdi ${ }^{*}$, Nourah M Aljameel ${ }^{1,2}$, Eyad F. Alsaeed ${ }^{3}$, Amina A. \\ Hassan $^{1}$ \\ ${ }^{1}$ Department of Clinical Laboratory Sciences, College of Applied Medical Sciences, King Saud University, Riyadh, \\ Kingdom of Saudi Arabia \\ ${ }^{2}$ MBBS, KSU Fellowship Clinical Chemistry, Kingdom of Saudi Arabia \\ ${ }^{3}$ King Saud University/ MBBs, FRCPC, Consultant Radiation Oncology, KSUMC, Riyadh, Kingdom of Saudi Arabia
}

\begin{abstract}
Objective: To determine the efficiency of granulocytes and monocytes upon radiotherapy in Saudi females with breast cancer.

Method: Samples from fifteen Saudi females with breast cancer and 30 control samples were included in this study. Functions of specific granulocytes and monocytes were examined by Phagotest, and data were analysed by FACS Calibur flow cytometer. These investigations were performed for control and patient groups after obtaining three different samples: pre-radiotherapy (pre-RT), immediately after completing the radiotherapy course, and six weeks following the completion of the radiotherapy course. Patients' biochemical tests including those for blood sugar, total bilirubin, alkaline phosphatase, aspartate transaminase, alanine transaminase, blood urea nitrogen, and creatinine levels, were assessed to evaluate their health status pre-RT. In addition, the correlation between each of the total doses and volume of an irradiated area with complete blood count (CBC) and the phagocytic functions of granulocytes and monocytes were studied.

Results: The following changes were observed: (1) at pre-RT baseline, the changes in $\mathrm{CBC}$ and monocytes' phagocytic activities were non-significant; significant decrease was found in granulocytes' phagocytic percentages and geographic mean (GMs); (2) at the end of RT, a significant reduction occurred in total white blood cell counts, platelets, lymphocytes count, and a significant increase in red blood cell counts, hemoglobin level, and granulocytes' phagocytic GMs; (3) after 6 weeks, changes in $\mathrm{CBC}$ and phagocytic activities of granulocytes and monocytes were non-significant.

Conclusion: Saudi breast cancer patients might be at hematological and immunological risk following RT. Therefore, the follow-up of such patients needs to include the assessment of phagocytic activities besides CBC.
\end{abstract}

Keywords: Breast cancer, Granulocytes, Monocytes, Radiotherapy, Phagocytic function, CBC.

Accepted on December 26, 2018

\section{Introduction}

In immunocompromised patients with cancer, chemotherapy and radiotherapy (RT) regimens, as well as, excessive administration of prophylactic and therapeutic antibiotics, have changed the epidemiology of infections. This contributes to the finding of infections as the major cause of morbidity and mortality, almost replacing the primary disease as the leading cause of death [1]. One of the premier lines of defense against infection is the activation of an innate immune system, such as polymorphonuclear leukocytes (PMNL) and monocytes, which play significant roles in maintaining the dynamic balance in the human immune system via phagocytosis [2]. Chemotherapy and RT lead to neutropenia and a general suppression of the body defense mechanisms. Severe functional impairment of granulocytes and monocytes increased the susceptibility to infection among neutropenic patients, as well as those with absent or marked neutropenia [3]. There was a widespread belief that only chemotherapy affects blood cell counts, and weakens the immune system, regardless of the RT effect. Although, the health hazards of X-ray on blood cell counts and physiological functions of PMNs among technicians working in this field have been conducted [4-6] in Saudi Arabia, investigations regarding the effect of RT on the counts and function of cancer patients' blood cells have not been addressed. Although many studies explored the effect of radiation on blood cell counts and functions according to their settings, their findings could not be generalized to Saudi 
patients. Saudi breast cancer female patients receive RT according to the American protocol, which disregards their genetic and environmental factors. This may increase the probability of protocol failure among some females. Therefore, assessments of granulocytes' efficiency in breast cancer patients during the pre- and post-RT periods are very critical to avoid a reduction in their function, which may save cancer patients' lives.

\section{Method}

\section{Subjects}

The present study included two groups; the first (cases) were 25 females aged 25 to $85 \mathrm{y}$ with histological diagnosis of stages I to IV breast cancer, selected from the Oncology Department of King Khalid University Hospital (KKUH). The second were 30 healthy women (controls), with matched age and socioeconomic characteristics with the first group. The controls were selected from the VIP clinic in KKUH, Riyadh, the Kingdom of Saudi Arabia (KSA). Twenty-five breast cancer patients who had undergone surgery and chemotherapy, and were scheduled to initiate a standard RT course, were invited to participate in this study between August 2016 and August 2017. Among both groups, females with history of repeated or current infection known to affect leukocyte counts and functions were excluded from the study. Patients enrolled in the study agreed to avoid taking any products containing known immune modulating compounds during the period of the study. Informed consent was obtained from each participant and the ethical approval was obtained from the institutional review board of KKUH. Three blood samples were collected from each patient, 1-before radiotherapy, 2-immediately after the completion of RT course, and 3-six-week post radiotherapy. However, some patients had poor compliance at follow-up. Hence, of these 25 cases screened at the pre-RT baseline, 16 completed the second follow-up, and 15 completed the third follow-up. Fifteen patients (cases) who completed all the 3 follow-ups were aged between 36 and $85 \mathrm{y}$. Twelve out of the 15 patients (cases) received neoadjuvant chemotherapy before irradiation. Docetaxel, cyclophosphamide, epirubicin and fluorouracil were the most commonly used chemotherapeutic agents. The hematopoietic drugs most frequently administered during the chemotherapy period were filgrastim and pegfilgrastim. The hormonal therapies that were used for the study participants (cases) were tamoxifen, letrozole, and femara (mainly before RT). A sample size of 12-15 was considered adequate to achieve significance in the description of inter-subject variability [7].

In this study, patients had an external beam of X-radiation from Varian linear accelerator in two medical centers. Thirteen of these patients (cases) had received the radiation in the RT department in King Fahad Medical City (KFMC), while two of them had received at King Faisal Specialist Hospital and Research Center (KFSHRC) in Riyadh. KFMC conveyed RT by using dual energy linear accelerator, which was applied using $3 \mathrm{D}$ conformal technique, and the dose ranged from
45-60 Gy/15-30 fractions, $5 \mathrm{~d}$ a week, for 5 to $6 \mathrm{w}$. Some patients (cases) who had wide local excision (WLE) of the breast with axillary lymph node dissection (ALND) type of surgeries had radiation boost of five extra sessions. While at KFSHRC, the RT was applied using True beam linear accelerator, with $3 \mathrm{D}$ conformal technique, and the dose ranged from 45-50 Gy/20-25 fractions $5 \mathrm{~d}$ a week, for 4 to $5 \mathrm{w}$.

Whole blood was obtained from both groups in EDTA- and sodium heparin anticoagulant-containing tubes. An EDTA tube was used for complete blood count (CBC) analysis along with the Beckman Coulter LH 780 (Beckman Coulter, Inc., United States) in the Hematology lab of KKUH, while the heparin tube was used for the granulocytes and monocytes' function test. The blood samples and the medical profile of patients were collected from the Oncology Department of KKUH, Riyadh, KSA. All patients' and controls' samples were assessed for CBC and specific granulocytes and monocytes' functions tests represented by Phagotest [8]. Also, biochemical data was collected from the patients' files for the evaluation of the patients health status before initiating RT. Biochemical data included blood sugar, total bilirubin, ALP, AST, ALT, BUN, and creatinine level.

The granulocytes and monocytes' phagocytic function were evaluated using the Phagotest kit. Phagotest was used to determine the monocytes and granulocytes' overall percentage, showing phagocytosis in general (ingestion of one or more bacteria), and the individual cellular phagocytic activity (the number of ingested bacteria per cell). The phagocytosis test kit contains fluorescein-labeled opsonized E. coli bacteria and other necessary reagents. Data acquisition was performed using FACS Calibur, Becton Dickenson (BD) (Cell Quest TM software).

\section{Statistical analysis}

Statistical analysis was performed using SPSS version 22.0 software (SPSS Inc., Chicago, IL, USA). Mean and standard deviation were calculated and reported for continuous variables. Student t-test for two independent groups was used to compare data between the control and patient (cases) groups before RT. Also, repeated measures ANOVA were used to compare the results before RT, immediately at the end of RT, and after $6 \mathrm{w}$. There was statistical difference if P-value $\leq 0.05$. Pearson's correlation coefficient was calculated, and the line regression analysis was fitted to determine the relationship between different parameters of $\mathrm{CBC}$, granulocytes and monocytes' phagocytic activities, and each of the total dose and volume of an irradiated area that received $100 \%$ of the dose.

\section{Results}

\section{Subjects}

Fifteen patients (cases) accomplished all the 3 follow-ups, at pre-RT baseline, immediately at the end of RT course, and after $6 \mathrm{w}$ RT completion. Table 1 shows the patients' clinical 
Efficiency of granulocytes and monocytes in breast cancer patients following radiotherapy in KSA

characteristics, while Table 2 shows their biochemical data at the pre-RT stage.

Table 1. The patients' clinical characteristics.

\begin{tabular}{|c|c|c|}
\hline Characteristics & Number (15) & $\%$ \\
\hline \multicolumn{3}{|l|}{ Stage: } \\
\hline 1 & 3 & $20 \%$ \\
\hline 2 & 0 & 0 \\
\hline 3 & 9 & $60 \%$ \\
\hline 4 & 3 & $20 \%$ \\
\hline \multicolumn{3}{|l|}{ Lymph nodes: } \\
\hline Negative & 2 & $13.30 \%$ \\
\hline Positive & 13 & $86.60 \%$ \\
\hline \multicolumn{3}{|l|}{ Site: } \\
\hline Right & 8 & $53.30 \%$ \\
\hline Left & 7 & $46.60 \%$ \\
\hline \multicolumn{3}{|l|}{ Estrogen/progesterone receptor: } \\
\hline Positive-positive & 10 & $66 \%$ \\
\hline Negative-negative & 2 & $13.30 \%$ \\
\hline Positive-negative & 3 & $20 \%$ \\
\hline \multicolumn{3}{|l|}{ HER 2/neu protein expression: } \\
\hline Positive & 1 & $6.67 \%$ \\
\hline Negative & 14 & 93.3 \\
\hline \multicolumn{3}{|l|}{ Cancer pathological type: } \\
\hline Invasive ductal carcinoma & 13 & $86.60 \%$ \\
\hline Invasive lobular carcinoma & 2 & $13.30 \%$ \\
\hline \multicolumn{3}{|l|}{ Surgery type: } \\
\hline Modified radical mastectomy (MRM) & 10 & $66 \%$ \\
\hline Wide local excision of breast (WLE) & 2 & $13.30 \%$ \\
\hline Wide local excision of breast (WLE)+axillary lymph node dissection (ALND) & 3 & $20 \%$ \\
\hline
\end{tabular}

Table 2. The biochemical data at the pre-radiotherapy stage.

\begin{tabular}{llll}
\hline Test & Mean + SD & Normal ranges & Percentage of normal range \\
\hline Blood sugar & $6.458 \pm 2.6145$ & $4.07-5.83 \mathrm{mmol} / \mathrm{L}$ & $8(53.33 \%)$ \\
\hline Total bilirubin & $6.371 \pm 2.366$ & $3-17$ Umol/L & $14(93.33 \%)$ \\
\hline Alkaline phosphatase (ALP) & $81.80 \pm 19.124$ & $40-150$ Unit/L & $15(100 \%)$ \\
\hline Aspartate transaminase (AST) & $18.20 \pm 7.939$ & $20-65$ Unit/L & $7(60 \%)$ \\
\hline Alanine transaminase (ALT) & $25.27 \pm 15.659$ & $2.5-6.4 \mathrm{mmol} / \mathrm{L}$ \\
\hline Blood urea nitrogen (BUN) & $4.44 \pm 1.559$ & $12(80 \%)$ \\
\hline
\end{tabular}




\section{Complete blood count parameters}

CBC data was displayed as mean \pm standard deviation. The differences in $\mathrm{CBC}$ parameters between both groups (control and cases) before exposure to RT were examined. In all $\mathrm{CBC}$ parameters, the means were lower than for controls except with eosinophils and basophils, which showed no significant differences between groups.

Furthermore, the blood picture among cases before and immediately at the end of RT was determined. There was a significant reduction in the mean total WBCs, platelets, and lymphocytes' counts among cases immediately after completing the RT course. However, there was a significant increase in RBCs and hemoglobin ( $\mathrm{Hgb}$ ) levels at the end of RT.

To examine the effect of RT after $6 \mathrm{w}$ RT completion, CBC parameters were measured. There was no significant alteration in CBC parameters after $6 \mathrm{w}$ RT completion compared to that measured immediately at the end of RT.

In order to demonstrate the effect of RT on CBC parameters whether it was reversible or not, differences between the means of CBC parameters in the control and patient (cases) groups after $6 \mathrm{w}$ RT completion were evaluated (Table 3). Notably, the analysis revealed that the percentage of hematocrit, platelets, and lymphocytes' counts had decreased significantly in comparison to that of the control group.

Table 3. Blood picture of controls and cases after $6 w$ RT completion.

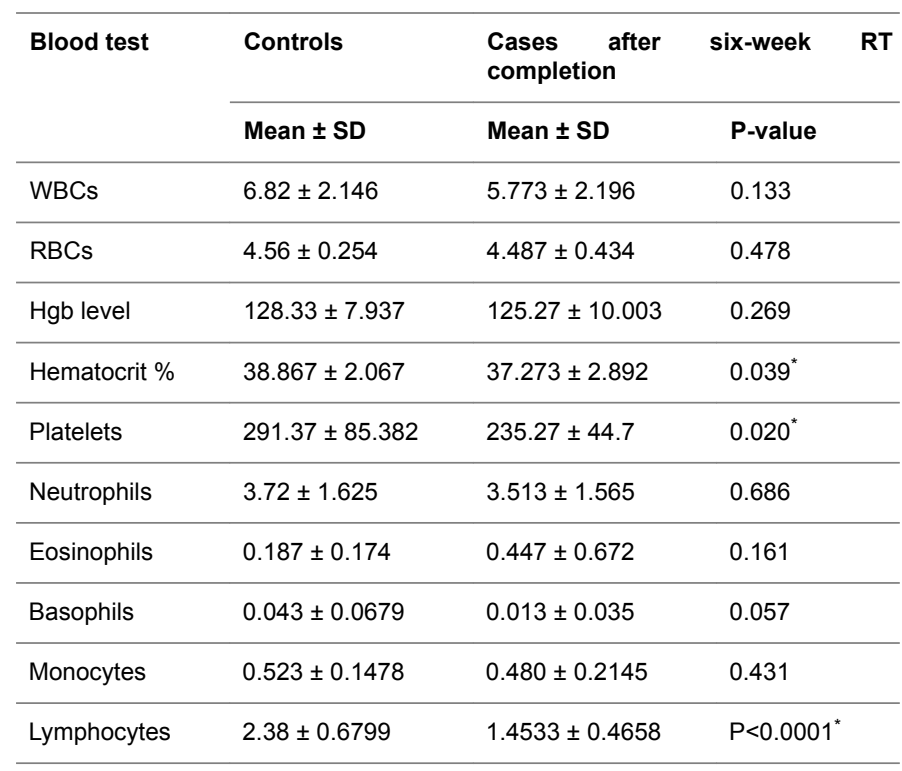

*Significant. A p-value $\leq 0.05$ was considered significant.

\section{Phagotest}

In each Phagotest analysis of control or patient sample, a negative control tube $\left(\right.$ at $0^{\circ} \mathrm{C}$ ) and a test tube (at $37^{\circ} \mathrm{C}$ ) were prepared to determine the quantitative phagocytic activity of granulocytes and monocytes. Then, all samples were read by FACS Calibur flow cytometer, and the data was analysed by the Cell Quest TM software program using Phagotest template and panels. A total of 10,000-15,000 leukocytes per sample was collected. An example of the granulocytes and monocytes' Phagotest measurement for a negative control tube (at $0^{\circ} \mathrm{C}$ ) for either control or patient sample are shown in Figures 1 and 2, respectively.
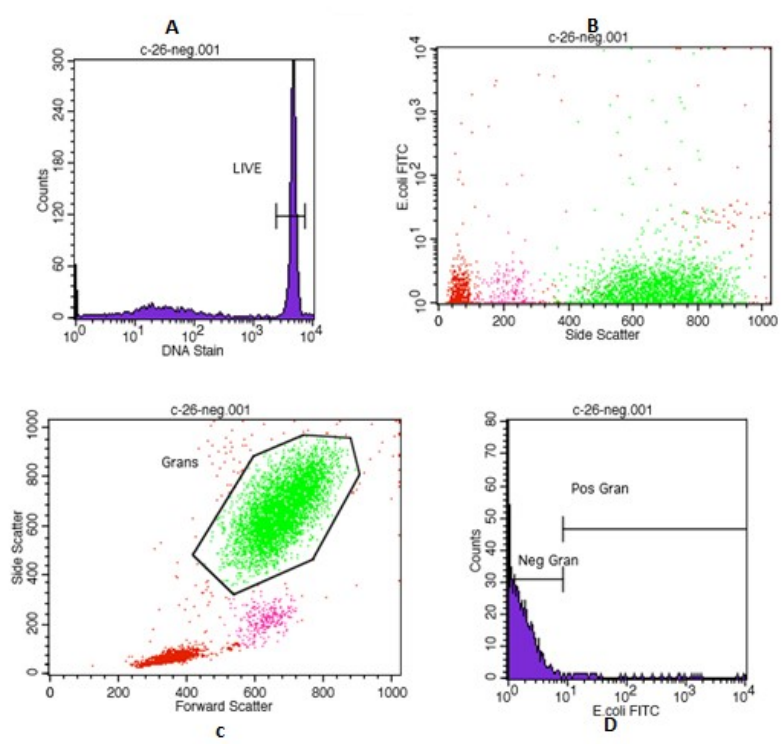

Figure 1. Quantitative determination of granulocytes phagocytic activity for a negative control tube $\left(\right.$ at $\left.0^{\circ} \mathrm{C}\right)$ for the control or patient sample. A: Red fluorescence (FL2) histogram displaying a live gate on leukocytes with at least the same DNA content as a human diploid cell (exclusion of bacteria aggregates) B: Dot plot showing the scatter light properties (lin side scatter (SSC)) vs. fluorescein isothiocyanate (FITC) fluorescence of labeled opsonized E. coli bacteria (log FL1). C: Typical dot plot lin forward scatter (FSC) vs. lin SSC with the relevant leukocyte cluster (i.e., granulocytes) was gated in the scatter diagram. D: Green fluorescence (FL1) of relevant leukocyte gate (i.e., granulocytes) was analysed using histogram analysis. 

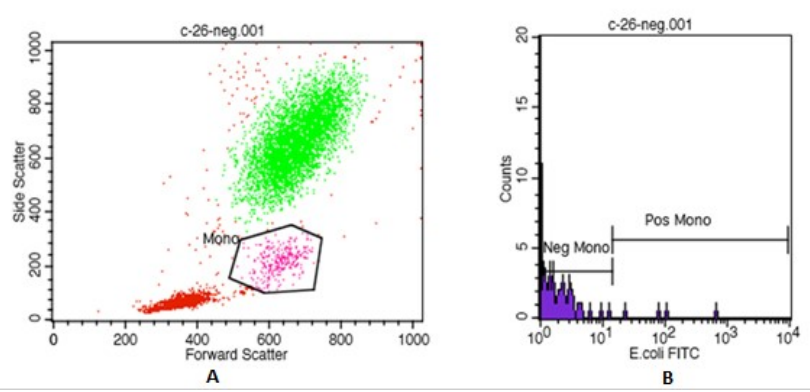

Figure 2. Quantitative determination of monocytes phagocytic activity for a negative control tube $\left(\right.$ at $\left.0^{\circ} \mathrm{C}\right)$ for the control or patient sample. A: Typical dot plot lin forward scatter (FSC) vs. lin side scatter (SSC) with the relevant leukocyte cluster (i.e., monocytes) was gated in the scatter diagram. B: Green fluorescence (FL1) of relevant leukocyte gate (i.e., monocytes) was analysed using histogram analysis.

Essentially, examples of granulocytes and monocytes' phagocytic activity measurements for a control sample (at $37^{\circ} \mathrm{C}$ ) as showed in Figures 3 and 4, respectively.
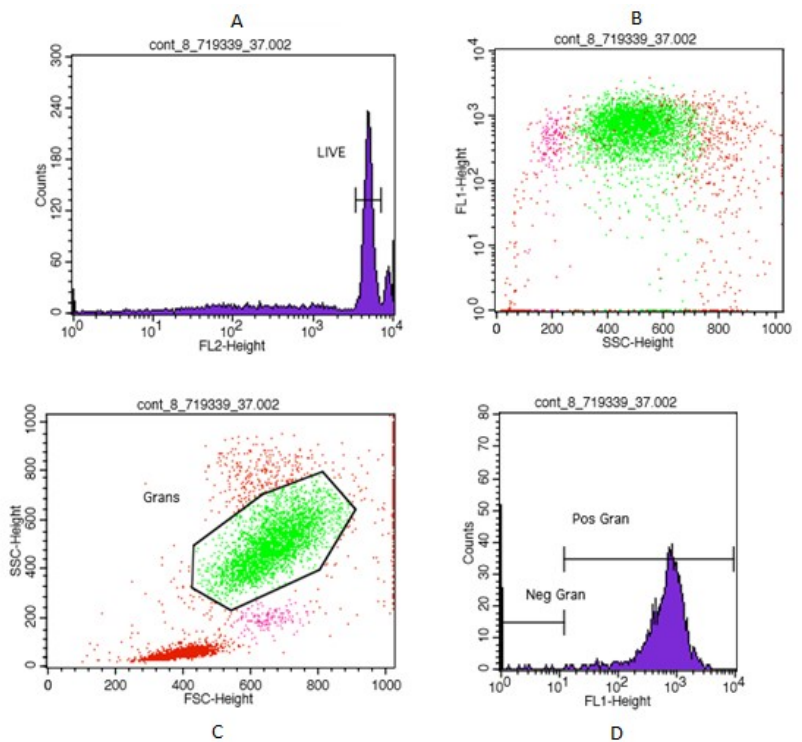

Figure 3. Quantitative determination of granulocytes phagocytic activity for a control sample test tube (at $\left.37^{\circ} \mathrm{C}\right)$. A: Red fluorescence (FL2) histogram displaying a live gate on leukocytes with at least the same DNA content as a human diploid cell (exclusion of bacteria aggregates) B: Dot plot showing the scatter light properties (lin side scatter (SSC)) vs. fluorescein isothiocyanate (FITC) fluorescence of labeled opsonized E. coli bacteria (log FL1). C: Typical dot plot lin forward scatter (FSC) vs. lin SSC with the relevant leukocyte cluster (i.e., granulocytes) was gated in the scatter diagram. D: Green fluorescence (FL1) of relevant leukocyte gate (i.e., granulocytes) was analysed using histogram analysis.
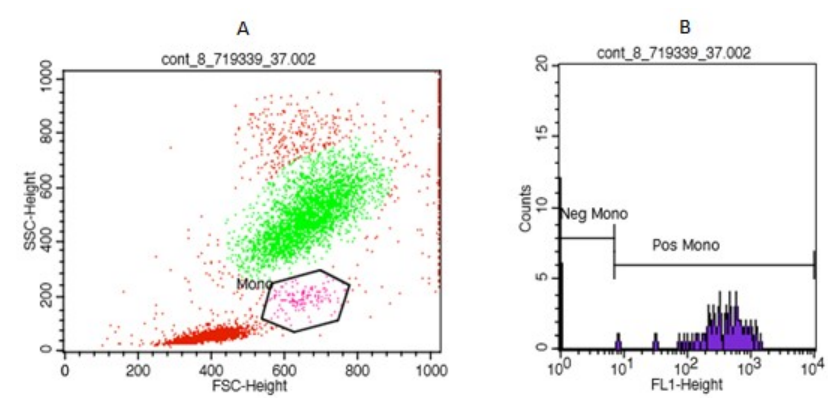

Figure 4. Quantitative determination of monocytes phagocytic activity for a control sample test tube $\left(\right.$ at $\left.37^{\circ} \mathrm{C}\right)$. A: Typical dot plot lin forward scatter (FSC) vs. lin side scatter (SSC) with the relevant leukocyte cluster (i.e., monocytes) was gated in the scatter diagram. B: Green fluorescence (FL1) of relevant leukocyte gate (i.e., monocytes) was analysed using histogram analysis.

Moreover, Figures 5 and 6 represent an example of granulocytes and monocytes phagocytic activity measurements for a patient sample $\left(\right.$ at $\left.37^{\circ} \mathrm{C}\right)$, respectively.
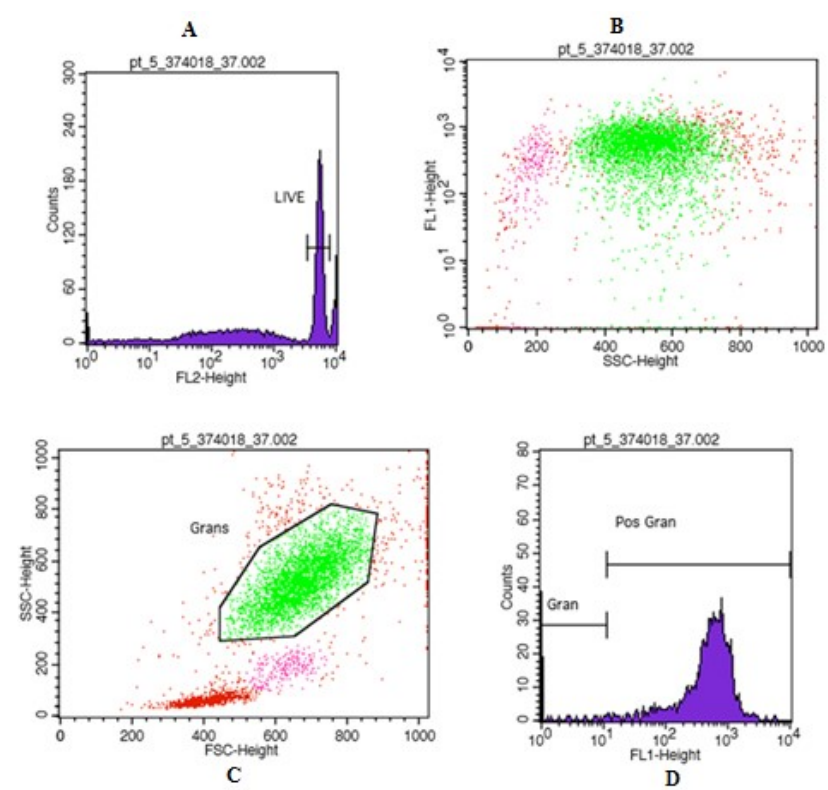

Figure 5. Quantitative determination of granulocytes phagocytic activity for a patient sample test tube (at $\left.37^{\circ} \mathrm{C}\right)$. A: Red fluorescence (FL2) histogram displaying a live gate on leukocytes with at least the same DNA content as a human diploid cell (exclusion of bacteria aggregates) B: Dot plot showing the scatter light properties (lin side scatter (SSC)) vs. fluorescein isothiocyanate (FITC) fluorescence of labeled opsonized E. coli bacteria (log FL1). C: Typical dot plot lin forward scatter (FSC) vs. lin SSC with the relevant leukocyte cluster (i.e., granulocytes) was gated in the scatter diagram. D: Green fluorescence (FL1) of relevant leukocyte gate (i.e., granulocytes) was analysed using histogram analysis. 

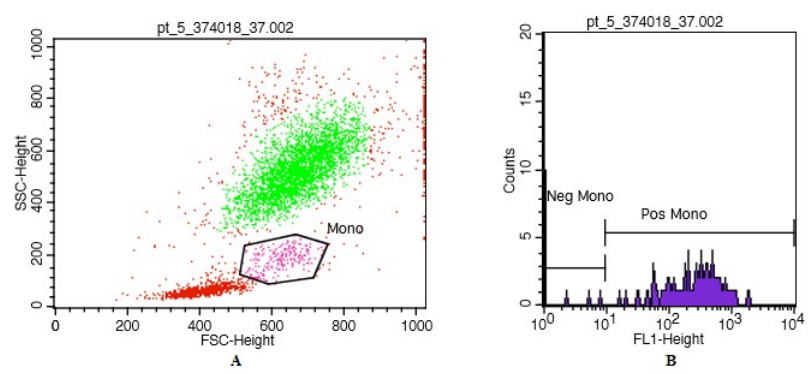

Figure 6. Quantitative determination of monocytes phagocytic activity for a patient sample test tube $\left(a t 37^{\circ} \mathrm{C}\right)$. A: Typical dot plot lin forward scatter (FSC) vs. lin side scatter (SSC) with the relevant leukocyte cluster (i.e., monocytes) was gated in the scatter diagram. B: Green fluorescence (FL1) of relevant leukocyte gate (i.e., monocytes) was analysed using histogram analysis.

With regards to granulocytes and monocytes' phagocytic activities, data were expressed as percentages of cells that executed phagocytosis, and as a number of ingested bacteria/ cell, where fluorescence intensity was expressed as geometrical mean (GM) for each cell type. The difference between granulocytes and monocytes' phagocytic activities of the 30 controls and 15 patient's pre-RT was studied. The means of cases' granulocytes phagocytic percentage and granulocytes GM were significantly lower than the means for the controls.

Particularly, a statistically significant increase in the mean of granulocytes' phagocytic GM occurred at the end of RT in comparison to that analysed before initiating RT.

There were no statistically significant changes in the means of granulocytes and monocytes phagocytic percentages and GM of cases after six weeks of RT.

To examine the reversibility of RT effects on granulocytes and monocytes' phagocytic functions, the means of granulocytes and monocytes' phagocytic function of controls were compared with that of cases after six-weeks of completing RT, Table 4. Remarkably, there were statistically significant reductions in granulocytes phagocytic percentage, granulocytes $\mathrm{GM}$, and monocytes phagocytic percentage.

Table 4. The means of granulocytes and monocytes phagocytic percentages and geographic mean among controls and cases after six-weeks of completing radiotherapy.

\begin{tabular}{llll}
\hline & Controls & Cases after six-weeks of completing RT & P-value \\
\cline { 1 - 2 } Cell function & Mean \pm SD & Mean \pm SD & $0.001^{*}$ \\
\hline Granulocytes phagocytic \% & $97.479 \pm 2.122$ & $90.936 \pm 9.105$ & $0.006^{*}$ \\
\hline Granulocytes GM & $743.647 \pm 284.378$ & $493.229 \pm 240.723$ & $0.024^{*}$ \\
\hline Monocytes phagocytic \% & $79.244 \pm 9.417$ & $71.949 \pm 10.718$ & 0.374 \\
\hline Monocytes GM & $358.543 \pm 184.58$ & $313.074 \pm 90.876$ & \\
\hline
\end{tabular}

The effect of each of the total dose of RT and the volume of an irradiated area that received $100 \%$ of the dose on the $\mathrm{CBC}$ parameters immediately at the end of RT was examined. A moderate negative relationship between eosinophils' counts immediately at the end of RT and the volume of an irradiated area was found, since Pearson's correlation coefficient $(r)=-0.570$ ( $P$ value, 0.026). Also, the same effect on the granulocytes and monocytes' phagocytic activities immediately at the end of RT were examined; a moderate positive relationship was observed between granulocytes' phagocytic percentage and total dose after six-weeks of RT, with $\mathrm{r}=0.595$ and $\mathrm{P}=0.019$. A moderate positive relationship between granulocytes phagocytic percentage and volume of an irradiated area after six weeks of RT was found, with $r=0.660$ and $\mathrm{P}=0.007$.

\section{Discussion}

The present investigation was designed to define the laboratory features, as well as the impact of RT on the role of granulocytes and monocytes function in breast cancer patients. All CBC parameters of the cases pre-RT showed non-statistical significant changes. This may be due to the colony stimulating factors (Filgrastim or Pegfilgrastim), which were given to patients, who were treated with chemotherapeutic agents to help make more WBCs. Importantly, a significant reduction in total WBCs, platelets, and lymphocyte counts were found at the end of RT, suggesting that these changes are related to a specific radiation effect on the immune cells. The decrease in the mean total WBCs count immediately following RT completion may be attributable to the short lifespan of leukocytes in peripheral blood (about $4 \mathrm{~d}$ for neutrophils, which comprise about $60 \%$ of leukocytes) [9]. Therefore, the harmful effect of radiation on the leukocytes' precursor would be reflected on the total leukocyte counts within a short time; because new ones from the hematopoietic tissues that were damaged by radiation will not be able to quickly replace a large number of damaged leukocytes in the peripheral blood. Moreover, the significant reduction in total WBCs may be explained by the slow recovery of radiation-damaged lymphocytes (which comprise about $30 \%$ of WBCs), due to a relatively long maturation phase of developing lymphocytes in the bone marrow and thymus, before their release into the peripheral blood [10]. In addition, a significant reduction was observed in the lymphocyte count after RT. The significant decrease in lymphocytes may explain the significant decrease in the mean total WBCs post-RT because lymphocytes comprise about $30 \%$ of WBCs. Also, this may be due to the 
sensitivity of these cells to radiation and bone marrow depression [11-13] and may be due to the slow recovery of radiation-damaged lymphocytes [10]. Also, granulocytes are generally more radiation resistant than lymphocytes [14]. The immune cells is known to be vulnerable to radiation, through induced apoptosis in mature T- and B-lymphocytes, and by lethal damage of stem cell precursors of monocytes and granulocytes in the bone marrow, as well as in natural killer cells [15].

In case of breast cancer, during the treatment period, radiation beams may induce the apoptosis of blood cells or hit parts of the sternum (ribs with clavicles), which have the maximum percentage of bone marrow contents, at 4.8 and 17.9, respectively [16]. The study data showed a significant increase in the mean RBCs count and Hgb level immediately at the end of RT. Bashir et al. reported that radiation with gamma or Xray, known to cause erythrocyte lysis, damage to the RBCs membrane, and increased membrane permeability, leading to potassium and hemoglobin leakage in the plasma [17]. The difference between means $\mathrm{CBC}$ of controls and cases after sixweeks of RT shows a statistically significant reduction in hematocrit percentage, platelets, and lymphocytes' counts of cases. These findings reflect that the effect of radiotherapy on $\mathrm{CBC}$ after six-weeks was not obvious, and reversible changes might occur, as there is no reduction in total WBCs, RBCs, $\mathrm{Hgb}$ level, neutrophils, eosinophils, and monocytes.

The effect of the disease and previous chemotherapy was clear on granulocytes' phagocytic activities among the cases, preRT, where there were high statistically significant decreases in granulocytes' phagocytic percentages, and granulocytes' GM. The significant increase in granulocytes' GM in breast cancer females immediately at the end of RT is in agreement with a study [18] which reported that the phagocytic indices of healthy rabbits as tested with Staphylococcus aureus increased significantly following radiation. The increase in phagocytosis may be explained by the fact that $46.67 \%$ of patients had an elevated blood sugar level than the normal range. Accordingly, they might have had diabetes, and the diabetes diet and treatment may increase phagocytosis as reported by Lecube et al. along with others [19]. Lecube et al. found significant negative correlations between phagocytic activity and fasting blood glucose $(\mathrm{r}=-0.619, \mathrm{p}=0.004)$, and HbA1c $(\mathrm{r}=-0.506, \mathrm{p}=$ 0.019 ) in type 2 diabetic patients. In addition, they found that patients who underwent metabolic optimization of diet and diabetes treatment showed a significant increase in phagocytic activity $(p=0.029)$. A decrease in the functional values of the desired cells was found, where granulocytes phagocytic percentages, granulocytes GM, and monocytes' phagocytic percentages values for cases after six-weeks significantly decreased. The granulocytes comprised the majority of these cells; thus, the effect of RT was irreversible until six-weeks after RT completion.

Traditionally, RT has been reported as an immunosuppressive agent, but many research published in recent years have suggested that the effects of RT on the immune system are complex. Locally administered RT can trigger systemic, abscopal, or 'out-of-field' effects. In the meantime, it is well known that response to DNA damage and the immunological events, such as anti-tumor immune mechanisms and inflammatory reactions are interconnected [20,21]. It worth noting that the RT is more likely to weaken the immune system if it is directed at the bones, especially the pelvic bones, where the bone marrow functions as a blood cell factory. With bone irradiation, the effect on the immune system can be similar to that of chemotherapy [22].

Additionally, it was found that there is no relationship between CBC parameters and the total dose of RT, and the volume of an irradiated area that received $100 \%$ of the dose, as the only correlation with eosinophils (at the end of RT), which represent very low percentage in the blood cells. While, a positive relationship of granulocytes' phagocytic percentage (which represent the majority of blood cells) with each of the total dose of RT, and the volume of an irradiated area was found. This positive relationship indicates that the function of these cells was affected more than their counts. Moreover, the linear regression analysis can be used to predict equations for future calculations of granulocytes phagocytic percentage, based on the value of the area and the total dose, as this correlation was significant.

\section{Conclusion}

This study was the first one in Saudi Arabia that described changes in multiple hematologic parameters including immune cell counts and functions in breast cancer patients pre- and post-RT. The impact of radiation on immune cells may be tumor type dependent and vary with the specificity of the used treatment protocol. Therefore, there is a persistent need for more research that will investigate the adjuvant therapy effect on Saudi breast cancer patients, to enhance the irradiation treatment efficacy, with the least unfavorable hematological and immunological effects. The results of this work strongly suggest that Saudi breast cancer female may be at hematological and immunological risk following RT, where the granulocytes and monocytes phagocytic activities of cases after six-weeks did not revert. However, the effect of RT was different for $\mathrm{CBC}$; while some items increased, others decreased non-significantly. Therefore, the follow-up of cancer patients must be with phagocytic activities besides $\mathrm{CBC}$, to readjust the cancer treatment plan or to prescribe medications such as antibiotics or growth factors that can stimulate blood cell production to avoid infections and other complications of cancer adjuvant therapy. Additionally, this study may be the first step in creating a suitable RT protocol for Saudi breast cancer females taking into consideration their genetic and environmental factors.

\section{Future Directions}

Future research should focus on increasing the sample size and extending the period of follow up. Also, all blood cell functions should be assessed. The observations from this study can assist in developing a suitable RT protocol for Saudi breast 
cancer females taking into consideration their genetic and environmental factors.

\section{References}

1. Koll B, Brown A. Changing patterns of infections in the immunocompromised patient with cancer. Hematol Oncol Clin N Am 1993; 7: 753-769.

2. Yona S, Jung S. Monocytes: subsets, origins, fates and functions. Curr Opin Hematol 2010; 17: 53-59.

3. Mayor S. Neutropenia in cancer patients: risk factors and management. Cancer World 2016; 71: 8.

4. Meo SA, Al Drees AM, Zadi SZ, Al Damgh S, AlTuwaijri AS. Hazards of X-ray radiation on the quantitative and phagocytic functions of polymorphonuclear neutrophils in X-ray technicians. J Occup Health 2006; 48: 88-92.

5. Meo SA. Hematological findings in male $x$-ray technicians. Saudi Med J 2004; 25: 852-856.

6. Meo SA. Phagocytic functions of polymorphonuclear neutrophils in dental $\mathrm{X}$-ray technicians. Pak Oral Dent J 2004; 24: 105-109.

7. Standish LJ, Torkelson C, Hamill FA, Yim D, Hill-Force A, Fitzpatrick A. Immune defects in breast cancer patients after radiotherapy. J Soc Integr Oncol 2008; 6: 110-121.

8. Glycotope Biotechnology GmbH. phagotest. Germany: Glycotope Biotechnology

9. 2014.

10. Tak T, Tesselaar K, Pillay J, Borghans JA, Koenderman L. Whats your age again? Determination of human neutrophil half-lives revisited. J Leukoc Biol 2013; 94: 595-601.

11. Awwad H. Radiation oncology: radiobiological and physiological perspectives: the boundary-zone between clinical radiotherapy and fundamental radiobiology and physiology: Springer Sci Business Media 2013.

12. West CM, Davidson SE, Elyan SA, Valentine H, Roberts SA, Swindell R. Lymphocyte radiosensitivity is a significant prognostic factor for morbidity in carcinoma of the cervix. Int J Radiat Oncol Biol Phys 2001; 51: 10-15.

13. Goans RE, Flynn DF. Acute radiation syndrome in humans. Medical consequences of radiological and nuclear weapons. Falls Church: Office of Surgeon General United States Army 2012: 17-38.
14. Heylmann D, Rodel F, Kindler T, Kaina B. Radiation sensitivity of human and murine peripheral blood lymphocytes, stem and progenitor cells. Biochim Biophys Acta 2014; 1846: 121-129.

15. Jacobs GP. A review on the effects of ionizing radiation on blood and blood components. Rad Phys Chem 1998; 53: 511-523.

16. Kusunoki Y, Hayashi T. Long-lasting alterations of the immune system by ionizing radiation exposure: implications for disease development among atomic bomb survivors. Int J Rad Biol 2008; 84: 1-14.

17. Hayman JA, Callahan JW, Herschtal A, Everitt S, Binns DS, Hicks RJ, Mac Manus M. Distribution of proliferating bone marrow in adult cancer patients determined using FLT-PET imaging. Int J Radiat Oncol Biol Phys 2011; 79: 847-852.

18. Bashir S, Naik F, Cardigan R, Thomas S. Effect of Xirradiation on the quality of red cell concentrates. Vox Sang 2011; 101: 200-207.

19. Rodel F, Keilholz L, Herrmann M, Sauer R, Hildebrandt G. Radiobiological mechanisms in inflammatory diseases of low-dose radiation therapy. Int $\mathrm{J}$ Rad Biol 2007; 83: 357-366.

20. Lecube A, Pachon G, Petriz J, Hernandez C, Simo R. Phagocytic activity is impaired in type 2 diabetes mellitus and increases after metabolic improvement. PloS One 2011; 6: 23366.

21. Formenti SC, Demaria S. Systemic effects of local radiotherapy. Lancet Oncol 2009; 10: 718-726.

22. Scheithauer $\mathrm{H}$, Belka $\mathrm{C}$, Lauber $\mathrm{K}$, Gaipl US. Immunological aspects of radiotherapy. Radiat Oncol 2014; 9: 185.

23. Breast cancer.org. How radiation therapy affects the immune system: Breastcancer.org; 2017.

*Correspondence to

Aminah A Alghamdi

Department of Clinical Laboratory Sciences

College of Applied Medical Sciences

King Saud University

Kingdom of Saudi Arabia 